nephron

Practice
Nephron 2018;140:99-104

DOI: $10.1159 / 000490119$
Received: May 8, 2018

Accepted after revision: May 16, 2018

Published online: May 31, 2018

\title{
Risk Prediction Models for Acute Kidney Injury in Critically III Patients: Opus in Progressu
}

\author{
Javier A. Neyra ${ }^{a}$ David E. Leaf ${ }^{b}$ \\ a Department of Internal Medicine, Division of Nephrology, Bone and Mineral Metabolism, University of Kentucky, \\ Lexington, KY, USA; ${ }^{b}$ Division of Renal Medicine, Brigham and Women's Hospital, Boston, MA, USA
}

\section{Keywords}

Acute Kidney Injury · Risk Prediction - Intensive care unit ·

Critical Illness

\begin{abstract}
Acute kidney injury (AKI) is a complex systemic syndrome associated with high morbidity and mortality. Among critically ill patients admitted to intensive care units (ICUs), the incidence of $\mathrm{AKI}$ is as high as $50 \%$ and is associated with dismal outcomes. Thus, the development and validation of clinical risk prediction tools that accurately identify patients at high risk for AKI in the ICU is of paramount importance. We provide a comprehensive review of 3 clinical risk prediction tools that have been developed for incident AKI occurring in the first few hours or days following admission to the ICU. We found substantial heterogeneity among the clinical variables that were examined and included as significant predic-
\end{abstract}

tors of $\mathrm{AKI}$ in the final models. The area under the receiver operating characteristic curves was $\sim 0.8$ for all 3 models, indicating satisfactory model performance, though positive predictive values ranged from only 23 to $38 \%$. Hence, further research is needed to develop more accurate and reproducible clinical risk prediction tools. Strategies for improved assessment of AKI susceptibility in the ICU include the incorporation of dynamic (time-varying) clinical parameters, as well as biomarker, functional, imaging, and genomic data.

(c) 2018 S. Karger AG, Basel

Contribution from the AKI and CRRT 2018 Symposium at the 23rd International Conference on Advances in Critical Care Nephrology, Manchester Grand Hyatt, San Diego, CA, USA, February 26 - March 1, 2018. This symposium was supported in part by the NIDDK funded University of Alabama at Birmingham-University of California San Diego O'Brien Center for Acute Kidney Injury Research (P30DK079337).

\section{KARGER}

(c) 2018 S. Karger AG, Basel

E-Mail karger@karger.com

www.karger.com/nef
Dr. Javier A. Neyra

Department of Internal Medicine, Division of Nephrology

University of Kentucky Medical Center

800 Rose St, MN668, Lexington, KY 40536 (USA)

E-Mail javier.neyra@uky.edu 


\section{Introduction}

Approximately 5 million patients are admitted to intensive care units (ICUs) in the United States each year, and as many as $50 \%$ of these patients are affected by acute kidney injury (AKI) [1-3]. AKI is a complex systemic syndrome associated with high morbidity, mortality, resource utilization, and risk for kidney and non-kidney related complications $[3,4]$. Survivors of AKI are at higher risk for the development or progression of chronic kidney disease (CKD) [5, 6], end-stage renal disease [7], and cardiovascular disease $[8,9]$. Early risk stratification for $\mathrm{AKI}$ in the ICU is challenging but is essential in developing novel strategies for AKI prevention and treatment [10]. In this article, we discuss the evolving field of clinical risk prediction tools for AKI in the ICU, including a review of existing models, as well as some of the challenges, limitations, and future directions for research in the field.

\section{What Has Been Done in Relation to Clinical Risk Prediction Tools for AKI in the ICU?}

Several investigators have developed and validated clinical risk prediction models for AKI among heterogeneous groups of critically ill patients admitted to the ICU. We focused on original manuscripts published during the last 10 years that included a sample size of more than 200 critically ill adults. Utilizing these criteria, we identified 3 studies (Table 1a, b).

Sapphire Cohort. Kashani et al. [11] conducted a prospective, multicenter cohort study in which they used a hypothesis-free approach to assess a panel of 340 candidate urinary and plasma biomarkers for the early detection of AKI among a heterogeneous group of mixed (medical and surgical) ICU patients. They found that urinary levels of tissue inhibitor of metalloproteinases 2 (TIMP2) and insulin-like growth factor-binding protein 7 (IGFBP7), which are markers of cell cycle arrest, were significant predictors of AKI. To assess the clinical utility of urinary TIMP2 and IGFBP7 in predicting AKI, they also developed a clinical model and assessed its performance both with and without inclusion of the urinary biomarkers. Thus, the study was not designed specifically to develop a clinical risk prediction tool for AKI per se.

The clinical model tested 16 parameters characterized as exposures (acute risk factors) and susceptibilities (demographics and chronic risk factors). Exposures were evaluated up to 5 days prior to study enrollment. The final model included 9 variables ( 4 acute and 5 chronic; Table 1b). AKI was defined as stage $\geq 2$ of the Kidney Disease: Improving Global Outcomes (KDIGO)-AKI criteria (SCr [serum creatinine] and urine output) [12]. The timeframe for $\mathrm{AKI}$ assessment was the first $12 \mathrm{~h}$ following study enrollment. This notably narrow window is a potentially significant limitation because many of the patients may have already been well on their way toward developing stage $\geq 2 \mathrm{AKI}$ at the time of enrollment. Nonetheless, the final clinical model (without the inclusion of the urinary biomarkers of interest) exhibited an area under the receiving operating characteristic curve (AUC) of 0.81 , with a $95 \%$ CI of $0.76-0.85$, for the prediction of AKI stage $\geq 2$ (Table 1a).

University of California San Diego-Mayo Cohort. Malhotra et al. [13] developed a clinical risk prediction model for AKI in the ICU using 2 independent cohorts: a discovery cohort from the University of California San Diego, and an external validation cohort from Mayo Clinic. They used forward elimination multiple logistic regression to select the variables for inclusion in their model and developed a scoring system using regression coefficients. Candidate continuous variables were dichotomized based on optimal cutoffs (e.g., blood pH $\leq 7.30$ ) using Youden's index method.

The final model included 10 clinical risk factors for AKI ( 5 acute and 5 chronic; Table $1 \mathrm{~b}$ ). All variables were evaluated within $48 \mathrm{~h}$ following ICU admission. Each risk factor was weighted by assigning a score of 0 (if absent) or 1,2 , or 3 points (if present). The minimum total score was 0 and the maximum total score was 21 points. AKI was defined as stage $\geq 1$ of the KDIGO-AKI SCr-based criteria [12]. The timeframe for the assessment of AKI was the first 7 days following study enrollment. The AUC for the discovery and external validation cohorts was 0.79 (95\% CI 0.69-0.88) and 0.81 (95\% CI 0.78-0.83), respectively, and the positive predictive value (PPV) was $23 \%$ for a risk score $\geq 5$ (Table 1a). Notable limitations of the study were the lack of access to comprehensive physiologic data other than mean arterial pressure $<70 \mathrm{~mm} \mathrm{Hg}$, the absence of oliguria and need for renal replacement therapy in the definition of AKI, and the utilization of SCr at the time of ICU admission as the baseline SCr when preICU SCr values were not available. The latter could have misclassified patients with ongoing AKI at the time of ICU admission as not having AKI.

The "AKI Predictor". Flechet et al. [14] conducted a retrospective study using the Early Parenteral Nutrition Completing Enteral Nutrition in Adult Critically Ill Patients (EPaNIC) database. They developed and validated a clinical risk stratification tool to predict AKI during the 
Table 1.

a. Comparison of clinical risk prediction models for AKI in the ICU

\begin{tabular}{|c|c|c|c|}
\hline & Kashani et al. [11], 2013 & Malhotra et al. [13], 2017 & Flechet et al. [14], 2017 \\
\hline Sample size & $n=728$ & $n=2,017$ & $n=4,760$ \\
\hline Single- or multicenter & Multicenter & Single-center & Multicenter \\
\hline Type of ICU & Mixed & Mixed & Mixed \\
\hline Validation cohort & No & External validation & Internal validation \\
\hline Physiologic data available & No & Yes (limited) & Yes \\
\hline Biochemical data available & Yes & Yes & Yes \\
\hline $\begin{array}{l}\text { Number of risk factors in final } \\
\text { model }\end{array}$ & 9 (4 acute; 5 chronic) & 10 (5 acute; 5 chronic) & 13 (10 acute; 3 chronic) \\
\hline $\begin{array}{l}\text { Timeframe for examination } \\
\text { of clinical variables }\end{array}$ & $\begin{array}{l}\text { Up to } 5 \text { days prior to study } \\
\text { enrollment }\end{array}$ & $\begin{array}{l}\text { Within } 48 \mathrm{~h} \text { following } \\
\text { ICU admission }\end{array}$ & $\begin{array}{l}\text { Preadmission; ICU admission; } \\
\text { day } 1\end{array}$ \\
\hline AKI definition & $\begin{array}{l}\uparrow S C r \geq 100 \% \text { in } 7 \text { day or } \\
\text { UOP }<0.5 \mathrm{~mL} / \mathrm{kg} / \mathrm{h} \times>12 \mathrm{~h}\end{array}$ & $\begin{array}{l}\uparrow S C r \geq 0.3 \mathrm{mg} / \mathrm{dL} \text { in } 48 \mathrm{~h} \\
\text { or } \geq 50 \% \text { in } 7 \text { day }\end{array}$ & $\begin{array}{l}\uparrow S C r \geq 0.3 \mathrm{mg} / \mathrm{dL} \text { in } 48 \mathrm{~h} \\
\text { or } \geq 50 \% \text { in } 7 \text { day }\end{array}$ \\
\hline $\begin{array}{l}\text { RRT included in AKI } \\
\text { definition }\end{array}$ & Yes & No & Yes \\
\hline $\begin{array}{l}\text { Timeframe for AKI } \\
\text { determination }\end{array}$ & $\begin{array}{l}12 \mathrm{~h} \text { from biospecimen } \\
\text { collection }\end{array}$ & 7 day from study enrollment & 7 day from ICU admission \\
\hline $\begin{array}{l}\text { Baseline SCr } \\
\text { determination }\end{array}$ & See below* & $\begin{array}{l}\text { Mean of all SCr measurements } \\
7-365 \text { day prior to admission }\end{array}$ & See below** \\
\hline $\begin{array}{l}\text { How missing baseline } \mathrm{SCr} \\
\text { was addressed }\end{array}$ & SCr at the time of enrollment & SCr at ICU admission utilized & SCr back-calculated ${ }^{* * *}$ \\
\hline Incident AKI events, $\%$ & 14.0 & 37.2 & 28.5 \\
\hline PPV & NA & $23 \%$ for risk score $\geq 5$ & $38 \%$ \\
\hline AUC & 0.81 & $\begin{array}{l}0.79 \text { (internal validation) } \\
0.81 \text { (external validation) }\end{array}$ & $\begin{array}{l}0.86-0.88 \text { (discovery) } \\
0.80-0.84 \text { (validation) }\end{array}$ \\
\hline
\end{tabular}

* If at least 5 SCr values were available, the median of all values from 6 months to 6 days prior to enrollment was used. Otherwise, the lowest $\mathrm{SCr}$ value in the 5 days prior to enrollment was used.

** The lowest SCr within 3 months prior to ICU admission was used for elective admissions, and the lowest SCr within 3 months to 1 week prior to ICU admission was used for patients transferred to the ICU from the emergency department.

*** Using an eGFR of $75 \mathrm{~mL} / \mathrm{min} / 1.73 \mathrm{~m}^{2}$ (MDRD equation).

AKI, acute kidney injury; AUC, area under the receiver operating curve; ED, emergency department; eGFR, estimated glomerular filtration rate; ICU, intensive care unit; MDRD, modification of diet in renal disease; NA, not assessed; PPV, positive predictive value; RRT, renal replacement therapy; SCr, serum creatinine; UOP, urine output.

first 7 days of ICU stay. The candidate variable selection was performed using a random forest machine-learning scheme using correlation-based ranking algorithms. Clinical parameters were evaluated at 3 time points in relation to ICU admission: pre-admission, ICU admission, and Day 1 of ICU stay. In addition, the investigators divided the dataset into development and validation cohorts.
The final variable selection was determined incrementally for each time-based model via bootstrapped backward elimination and included 5 variables with data available at pre-admission, 3 variables with data available on ICU admission, and 5 variables examined during the first day (Day 1) of ICU stay (Table 1b). AKI was defined as stage $\geq 1$ of KDIGO-AKI SCr-based criteria [12]. The timeframe for assessment of AKI was the first 7 days fol- 
Table 1. (continued)

b. Variables included in clinical risk prediction models for AKI in the ICU: comparison across models

\begin{tabular}{|c|c|c|c|}
\hline & Kashani et al. [11], 2013 & Malhotra et al. [13], 2017 & Flechet et al. [14], 2017 \\
\hline \multicolumn{4}{|l|}{ Demographics and comorbidities } \\
\hline \multicolumn{4}{|l|}{$\operatorname{Age}^{\mathrm{a}}$} \\
\hline \multicolumn{4}{|l|}{ Race } \\
\hline \multicolumn{4}{|l|}{$\mathrm{BMI}>30 \mathrm{~kg} / \mathrm{m}^{2}$} \\
\hline \multicolumn{4}{|l|}{ Baseline SCr } \\
\hline \multicolumn{4}{|l|}{ Hypertension } \\
\hline \multicolumn{4}{|l|}{ Congestive heart failure } \\
\hline \multicolumn{4}{|l|}{ Chronic liver disease } \\
\hline \multicolumn{4}{|l|}{ Chronic lung disease } \\
\hline \multicolumn{4}{|l|}{ Cardiovascular disease } \\
\hline \multicolumn{4}{|l|}{ Planned vs. unplanned ICU admission } \\
\hline \multicolumn{4}{|l|}{ Major surgery } \\
\hline \multicolumn{4}{|l|}{ Sepsis } \\
\hline \multicolumn{4}{|l|}{ Burns or trauma } \\
\hline \multicolumn{4}{|l|}{ Mechanical ventilation } \\
\hline \multicolumn{4}{|l|}{ Serum albumin } \\
\hline \multicolumn{4}{|l|}{ Anemia $^{c}$} \\
\hline \multicolumn{4}{|l|}{ Nephrotoxin exposure ${ }^{\mathrm{d}}$} \\
\hline \multicolumn{4}{|l|}{ IV or IA radiocontrast exposure } \\
\hline \multicolumn{4}{|l|}{ Blood $\mathrm{pH} \leq 7.30$} \\
\hline \multicolumn{4}{|l|}{ Blood glucose on ICU admission } \\
\hline
\end{tabular}

Blue and red boxes indicate variables that were assessed and were (blue) or were not (red) significant predictors of AKI in the final model; white boxes indicate that the variable was not assessed in the study. "X" denotes that the clinical parameter was included in the final model. " Based on "day 1 model" only.

a Age $>70$ years (Malhotra et al. [13]); used as a continuous variable in the other 2 studies.

${ }^{b}$ Recorded from the patient's medical history (Kashani et al. [11]); defined as eGFR $<60 \mathrm{~mL} / \mathrm{min} / 1.73 \mathrm{~m}^{2}$, calculated using the CKD epidemiology collaboration equation (Malhotra et al. [13]).

${ }^{c}$ Hematocrit $<30 \%$ (Kashani et al. [11]); Hemoglobin $<9 \mathrm{mg} / \mathrm{dL}$ or hematocrit $<27 \%$ (Malhotra et al. [13]).

${ }^{\mathrm{d}}$ Exposure to any of the following drugs within 5 days prior to and including the day of enrollment: NSAIDs, ACE inhibitors, Angiotensin II receptor blockers, calcineurin inhibitors, beta-lactam antibiotics, aminoglycosides, vancomycin, acyclovir, amphotericin, allopurinol, colistin (Kashani et al. [11]); exposure within 7 days prior to ICU admission to IV or IA radiocontrast, $\geq 3$ nephrotoxins (amphotericin, aminoglycosides, chemotherapy, anti-retroviral drugs, or NSAIDs), or $\geq 3$ days of aminoglycosides (Malhotra et al. [13]); exposure to nephrotoxins as a category was not evaluated by Flechet et al. [14], but the following individual medication exposures during the first day of ICU stay were examined, and none was a significant predictor of AKI in the final model: vancomycin, aminoglycosides, anti-retrovirals, beta-lactam antibiotics, diuretics, calcineurin inhibitors, antifungals, NSAIDs, ACE inhibitors, and IV or IA radiocontrast.

${ }^{\mathrm{e}}$ Hypotension (MAP $<70 \mathrm{~mm} \mathrm{Hg}$ ) within 5 days prior to and including the day of enrollment (Kashani et al. [11]); MAP <70 mm $\mathrm{Hg}$ or use of any vasopressor (Malhotra et al. [13]); mechanical or pharmacological hemodynamic support (Flechet et al. [14]).

APACHE, Acute Physiologic Assessment and Chronic Health Evaluation; BMI, body mass index; CKD, chronic kidney disease; HIV, human immunodeficiency virus; IA, intra-arterial; ICU, intensive care unit; IV, intravenous; SCr, serum creatinine. 
Fig. 1. Framework for implementation of biomarker testing into a clinical risk prediction tool for AKI in the ICU. AKI, acute kidney injury; ICU, intensive care unit; ACE-I, angiotensin-converting enzyme inhibitor; ARB, angiotensin II receptor blocker; KDIGO, Kidney Disease: Improving Global Outcomes; RCTs, randomized controlled trials; SCr, serum creatinine; UOP, urine output.

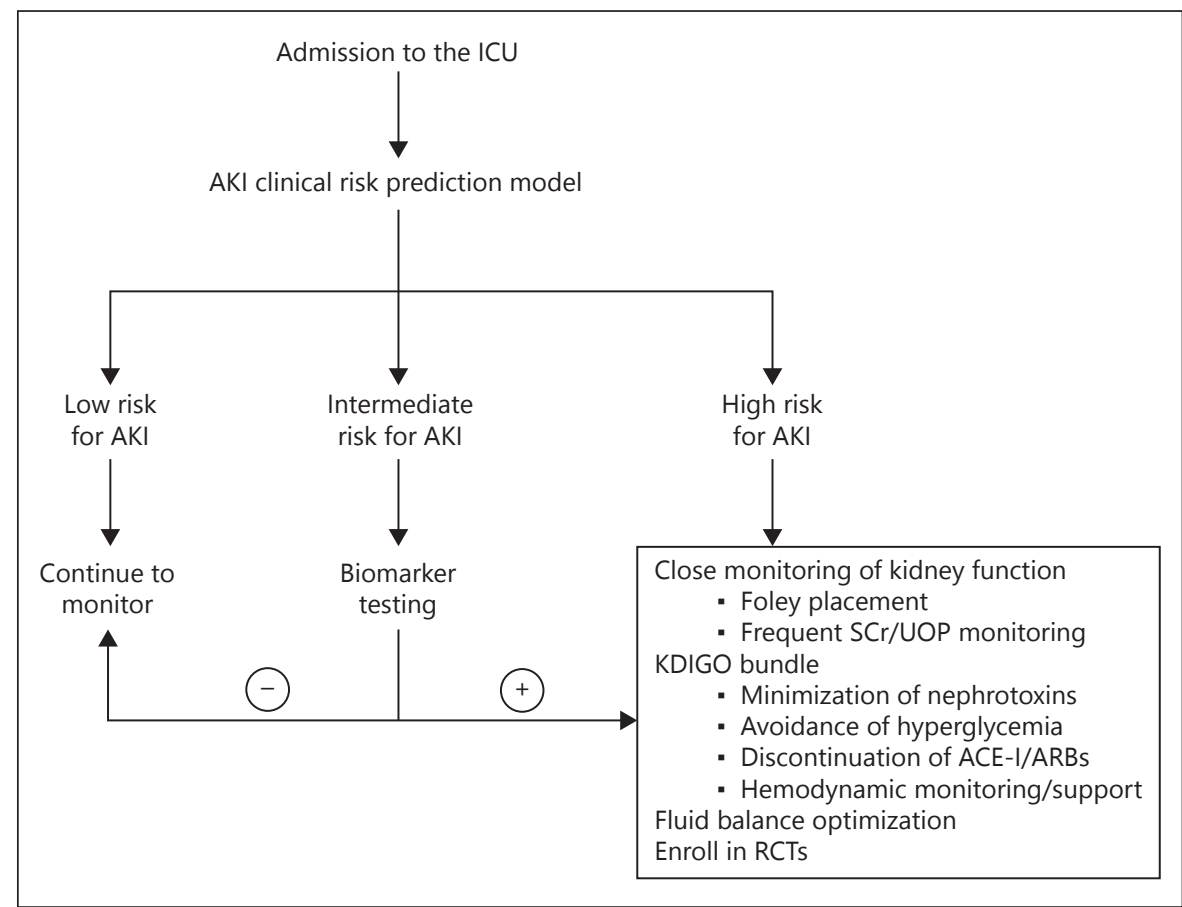

lowing ICU admission. The AUCs for the discovery cohort were 0.86 (95\% CI 0.86-0.86) and 0.88 (95\% CI 0.880.89 ) for AKI stage $\geq 1$ and $\geq 2$ respectively. These results were internally validated with repeated AUCs $\geq 0.80$ (Table $1 \mathrm{~b})$. The reported PPV of this clinical risk prediction tool was $38 \%$. Important limitations of this study were the absence of oliguria criterion in the AKI diagnosis and the assumption of a baseline estimated glomerular filtration rate of $75 \mathrm{~mL} / \mathrm{min} / 1.73 \mathrm{~m}^{2}$ for patients with missing baseline SCr value.

\section{Comparison of Clinical Risk Prediction Models}

Table la summarizes the design characteristics, definitions, and performance metrics among the 3 studies. Table $1 \mathrm{~b}$ shows a comparison of the variables included in each of the models. As demonstrated, the only variables that replicated across more than 1 model were $\mathrm{CKD}$, sepsis, and exposure to nephrotoxins, and no variable replicated across all 3 studies. Further, even among the variables that predicted AKI in more than 1 study, definitions used for these variables differed from study to study. For example, CKD was determined by Kashani et al. [11] based on the patient's medical history, while it was defined as baseline estimated glomerular filtration rate $<60 \mathrm{~mL} / \mathrm{min} / 1.73 \mathrm{~m}^{2}$ by Malhotra et al. [13] In con- trast, Flechet et al. [14] used baseline SCr rather than CKD in their risk prediction model. Similarly, the definition of nephrotoxin exposure varied widely across studies (Table $1 \mathrm{~b}$ ). Finally, many variables that predicted AKI in 1 study were simply not assessed by other studies.

\section{What Needs to be Done to Improve Risk Prediction Tools for AKI in the ICU?}

- Development of a large, heterogeneous, multicenter, and multiethnic cohort to refine, homogenize, and validate current clinical risk prediction models.

- Utilize advances in computer science and clinical informatics to incorporate dynamic (time-varying) clinical parameters that may be more informative for clinical risk prediction models than solely static clinical parameters.

- Incorporation of novel biomarkers of AKI into clinical risk prediction models. A suggested framework is provided in Figure 1.

- Incorporation of functional studies (e.g., furosemide stress test), imaging studies, and genomic data into clinical risk prediction models for more individualized and precise detection of AKI susceptibility.

- Development of risk stratification tools for the prognostication of AKI recovery. 
- Development of clinical decision support systems that enhance health-related decisions and timely interventions to prevent or attenuate AKI or promote its recovery.

- Strengthening of multidisciplinary collaborations among investigators from all latitudes, incorporating computer, bench, and clinical scientists.

\section{Conclusions}

It is essential to develop accurate and precise clinical risk prediction tools for AKI in the ICU, for timely intervention that can prevent or mitigate AKI and for enrichment of patients in randomized controlled trials. We reviewed 3 existing AKI risk prediction tools and found substantial heterogeneity in the variables that were included in the final models. Further, although all models had satisfactory AUCs, PPV ranged only from 23 to $38 \%$. Thus, additional research is needed to further validate, harmonize, and expand on existing clinical risk prediction tools for AKI in the ICU.

\section{Acknowledgments}

J.A.N. is supported by an Early Career Pilot Grant from the National Center for Advancing Translational Sciences, National Institutes of Health, through Grant UL1TR001998. D.E.L. is supported by the following grants from the National Institute of Diabetes and Digestive and Kidney Diseases: K23DK106448 and P30DK079337 (pilot and feasibility award from the University of Alabama at Birmingham-University of California, San Diego O'Brien Center for AKI Research). D.E.L. is also supported by an American Society of Nephrology Foundation for Kidney Research Carl W. Gottschalk Research Scholar Grant.

\section{Disclosure Statement}

The authors declare that they have no conflicts of interest to disclose.

\section{References}

1 Mandelbaum T, Scott DJ, Lee J, et al: Outcome of critically ill patients with acute kidney injury using the acute kidney injury network criteria. Crit Care Med 2011;39:26592664.

2 Neyra JA, Manllo J, Li X, et al: Association of de novo dipstick albuminuria with severe acute kidney injury in critically ill septic patients. Nephron Clin Pract 2014;128:373-380.

-3 Waikar SS, Liu KD, Chertow GM: Diagnosis, epidemiology and outcomes of acute kidney injury. Clin J Am Soc Nephrol 2008;3:844861.

4 Lafrance JP, Miller DR: Acute kidney injury associates with increased long-term mortality. J Am Soc Nephrol 2010;21:345-352.

5 Chawla LS, Amdur RL, Amodeo S, Kimmel PL, Palant CE: The severity of acute kidney injury predicts progression to chronic kidney disease. Kidney Int 2011;79:1361-1369.
6 Coca SG, Singanamala S, Parikh CR: Chronic kidney disease after acute kidney injury: a systematic review and meta-analysis. Kidney Int 2012;81:442-448.

7 Wald R, Quinn RR, Adhikari NK, et al: Risk of chronic dialysis and death following acute kidney injury. Am J Med 2012;125:585-593.

-8 Wu VC, Wu CH, Huang TM, et al: Long-term risk of coronary events after AKI. J Am Soc Nephrol 2014;25:595-605.

-9 Bansal N, Matheny ME, Greevy RA Jr, et al. Acute kidney injury and risk of incident heart failure among US veterans. Am J Kidney Dis 2018;71:236-245.

10 Meersch M, Schmidt C, Hoffmeier A, et al: Prevention of cardiac surgery-associated AKI by implementing the KDIGO guidelines in high risk patients identified by biomarkers: the PrevAKI randomized controlled trial. Intensive Care Med 2017;43:1551-1561.
11 Kashani K, Al-Khafaji A, Ardiles T, et al: Discovery and validation of cell cycle arrest biomarkers in human acute kidney injury. Crit Care 2013;17:R25.

12 Kidney Disease: Improving Global Outcomes (KDIGO) acute kidney injury work group. KDIGO clinical practice guideline for acute kidney injury. Kidney Int Suppl 2012;2:1138.

13 Malhotra R, Kashani KB, Macedo E, et al. A risk prediction score for acute kidney injury in the intensive care unit. Nephrol Dial Transplant 2017;32:814-822.

14 Flechet M, Guiza F, Schetz M, et al. AKIpredictor, an online prognostic calculator for acute kidney injury in adult critically ill patients: development, validation and comparison to serum neutrophil gelatinase-associated lipocalin. Intensive Care Med 2017;43:764773. 